\title{
Alternative activation of human macrophages enhances tissue factor expression and pro- duction of extracellular vesicles
}

Haematologica 2021

Volume 106(2):454-463

\section{Correspondence:}

JOHANN WOJTA

johann.wojta@meduniwien.ac.at

Received: February 26, 2019.

Accepted: January 23, 2020.

Pre-published: January 23, 2020.

https://doi.org/10.3324/haematol.2019.220210

(C)2021 Ferrata Storti Foundation

Material published in Haematologica is covered by copyright. All rights are reserved to the Ferrata Storti Foundation. Use of published material is allowed under the following terms and conditions:

https://creativecommons.org/licenses/by-nc/4.0/legalcode. Copies of published material are allowed for personal or internal use. Sharing published material for non-commercial purposes is subject to the following conditions:

https://creativecommons.org/licenses/by-nc/4.0/leǵalcode, sect. 3. Reproducing and sharing published material for commercial purposes is not allowed without permission in writing from the publisher.

\author{
Philipp J. Hohensinner,,${ }^{1,2}$ Julia Mayer, ${ }^{1}$ Julia Kirchbacher, ${ }^{1}$ Julia Kral-Pointner, ${ }^{1,2}$ \\ Barbara Thaler, ${ }^{1}$ Christoph Kaun, ${ }^{1}$ Lena Hell, ${ }^{3}$ Patrick Haider, ${ }^{1}$ Marion \\ Mussbacher, ${ }^{4}$ Johannes A. Schmid, ${ }^{4}$ Stefan Stojkovic, ${ }^{1}$ Svitlana Demyanets, ${ }^{1,5}$ \\ Michael B. Fischer, ${ }^{6,7}$ Kurt Huber,,${ }^{2,8,9}$ Katharina Wöran, ${ }^{10}$ Christian \\ Hengstenberg, ${ }^{1}$ Walter S. Speidl, ${ }^{1}$ Rudolf Oehler, ${ }^{11}$ Ingrid Pabinger ${ }^{3}$ and Johann \\ Wojta ${ }^{1,2,12}$
}

${ }^{1}$ Department of Internal Medicine II/Cardiology, Medical University of Vienna, Vienna; ${ }^{2}$ Ludwig Boltzmann Institute for Cardiovascular Research, Vienna; ${ }^{3}$ Department of Internal Medicine I, Medical University of Vienna, Vienna; ${ }^{4}$ Institute of Vascular Biology and Thrombosis Research, Medical University of Vienna, Vienna; ${ }^{5}$ Department of Laboratory Medicine, Medical University of Vienna, Vienna; ${ }^{6} \mathrm{Clinic}$ for Blood Group Serology and Transfusion Medicine, Medical University of Vienna, Vienna; ${ }^{7}$ Department for Health Science and Biomedicine, Danube University Krems, Krems; ${ }^{8}{ }^{\text {rd }}$ Medical Department, Wilhelminenhospital, Vienna; ${ }^{9}$ Medical Faculty, Sigmund Freud University, Vienna; ${ }^{10}$ Department of Pathology, Medical University of Vienna, Vienna; ${ }^{11}$ Department of Surgery, Medical University of Vienna, Vienna and ${ }^{12}$ Core Facilities, Medical University of Vienna, Vienna, Austria

\section{ABSTRACT}

M acrophages are versatile cells that can be polarized by the tissue environment to fulfill required needs. Proinflammatory polarization is associated with increased tissue degradation and propagation of inflammation whereas alternative polarization within a Th2 cytokine environment is associated with wound healing and angiogenesis. To understand whether polarization of macrophages can lead to a procoagulant macrophage subset we polarized human monocyte-derived macrophages to proinflammatory and alternative activation states. Alternative polarization with interleukin-4 and interleukin-13 led to a macrophage phenotype characterized by increased tissue factor (TF) production and release and by an increase in extracellular vesicle production. In addition, TF activity was enhanced in extracellular vesicles of alternatively polarized macrophages. This TF induction was dependent on signal transducer and activator of transcription-6 signaling and poly ADP ribose polymerase activity. In contrast to monocytes, human macrophages did not show increased TF expression upon stimulation with lipopolysaccharide and interferon- $\gamma$. Previous polarization to either a proinflammatory or an alternative activation subset did not change the subsequent stimulation of TF. The inability of proinflammatory activated macrophages to respond to lipopolysaccharide and interferon$\gamma$ with an increase in TF production seemed to be due to an increase in TF promoter methylation and was reversible when these macrophages were treated with a demethylating agent. In conclusion, we provide evidence that proinflammatory polarization of macrophages does not lead to enhanced procoagulatory function, whereas alternative polarization of macrophages leads to an increased expression of TF and increased production of TF-bearing extracellular vesicles by these cells suggesting a procoagulatory phenotype of alternatively polarized macrophages.

\section{Introduction}

Macrophages are cells of the innate immune system which play numerous and vastly different functions within the body. Macrophages reside in all tissues of the body and each population of macrophages within a tissue can take on specialized functions that are tuned to the developmental and functional requirements of that 
tissue. ${ }^{1}$ Some tissue macrophages and precursors are already established embryonically in the yolk sac and fetal liver before the onset of definitive hematopoiesis. ${ }^{2}$ Upon inflammation, the pool of resident macrophages gets quickly replaced by macrophages derived from circulating monocytes. ${ }^{3}$

In order to be able to respond to multiple tasks, macrophages can adapt to the environment when exposed to specific cues. This macrophage polarization can be simulated in vitro using lipopolysaccharide (LPS) and interferon (IFN)- $\gamma$ stimulation for a proinflammatory subset termed classical activation and stimulation with interleukin (IL)-4 and IL-13 for an alternative polarization phenotype. ${ }^{4}$ Upon polarization, macrophages react to the respective stimulus with the expression of a distinct phenotype. Classical polarization is usually associated with a proinflammatory response, including the increased production of tumor necrosis factor (TNF)- $\alpha$, IL-1, and IL- $6 .{ }^{5}$ Functionally, proinflammatory polarization leads to potent effector cells that kill intracellular micro-organisms and tumor cells. ${ }^{6}$ In addition, these cells are present during early wound healing and proinflammatory macrophages are characterized by a pronounced ability to degrade tissue. ${ }^{7}$ In contrast, alternatively activated macrophages are characterized by increased expression of IL-10 and of scavenger receptors. Besides scavenging debris, promoting angiogenesis, tissue remodeling and repair, alternatively activated macrophages are able to fine tune inflammatory responses. ${ }^{8}$ In vivo, distinct macrophage subtypes are more subtle. Nevertheless, in vitro polarization phenotypes are present in in vivo situations. ${ }^{4}$ Macrophages resembling classical macrophages can be found in environments with bacterial infection or in inflammatory pathologies, whereas alternatively polarized macrophages are prominent in cancer and in diseases with a Th2 cytokine signature. ${ }^{9}$

An inflammatory milieu is usually accompanied by an increased procoagulatory risk. This risk increase is due to the close link between inflammation and tissue factor (TF) induction. TF is the primary activator of the coagulation cascade. ${ }^{10}$ TF-bearing monocytes are essential for the initiation of coagulation ${ }^{11}$ and TF expression in monocytes is triggered strongly by inflammatory mediators including LPS. ${ }^{10}$ Nonetheless, whereas TF expression in human monocytes is well studied, data on TF production in human macrophages, especially under different polarization conditions, are scarce. TF is a transmembrane protein that functions as a high affinity receptor for factor VII and the activated form of this clotting factor. ${ }^{10}$ Especially in circulation, TF is present on circulating extracellular vesicles. In pathological conditions, high levels of procoagulant, TFexposing particles can be found in the circulation. ${ }^{12}$ Interestingly, even though TF and inflammation are closely connected, cancer and hence a Th2-promoting environment were also found to be associated with increased TFbearing extracellular vesicles. ${ }^{13}$ Extracellular vesicles are membrane-enclosed structures of different sizes. ${ }^{14}$ TF is mainly associated with vesicles within the microvesicle fraction of extracellular vesicles which range in size from 200-1000 nm..$^{15}$ These vesicles form through an active budding mechanism from the parental cell and, therefore, include membrane-linked proteins. ${ }^{16}$ In addition to TF those vesicles can have phosphatidylserine exposed on the surface which, in turn, can both support coagulation ${ }^{17}$ and activate $\mathrm{TF}^{10}$
In order to understand a possible role of macrophages in fostering a procoagulatory environment in different disease states, we investigated the contribution of macrophages and their respective polarization to the production and release of TF and of extracellular vesicles.

\section{Methods}

Full details of the experimental setups and conditions are available in the Online Supplement.

\section{Generation of human monocytes and macrophages}

Human macrophages were derived from peripheral blood monocytes from leukapheresis chambers, as described previously, obtained from healthy platelet donors according to recommendations of the ethical board of the Medical University of Vienna including informed consent (approval number 1575/2014), ${ }^{7,18}$ Human macrophages were always prepared from fresh blood. Macrophages from 75 healthy volunteers were used in the course of the study.

\section{Flow cytometry}

Extracellular vesicles were analyzed using flow cytometry on a Cytoflex (Beckman Coulter, CA, USA) or AttuneNXT (Thermo Fisher, MA, USA) flow cytometer. Values are given as events/ $\mathrm{L}$. Microvesicles were defined as being between $200 \mathrm{~nm}$ and $900 \mathrm{~nm}$ according to size-specific fluorescence beads (Megamix Plus, Biocytex, France). Human and mouse monocytes were investigated for membrane-bound TF using a specific antibody (Thermo Fisher [MA, USA] for human TF, Biotechne [MN, USA] for mouse TF) as published previously. ${ }^{19}$

\section{Enzyme-linked immunosorbent assay of extracellular vesicles}

We used a commercially available enzyme-linked immunosorbent assay (ELISA) kit (Hyphen Biomed, France) to determine the concentration of annexin $\mathrm{V}^{+}$extracellular vesicles in the circulation according to the manufacturer's instructions.

\section{RNA isolation and quantitative polymerase chain reaction analysis}

Detailed information about RNA isolation and quantitative polymerase chain reactions are available in the Online Supplement.

\section{Protein determination}

TF was determined by a commercially available ELISA (Biotechne, MN, USA) as suggested by the manufacturer on extracellular vesicles and in cell lysates. Extracellular vesicles were isolated by centrifugation at $18,000 \mathrm{~g}$ for $20 \mathrm{~min}$ at $4^{\circ} \mathrm{C}$

\section{Tissue factor activity assay}

Extracellular vesicle-associated TF activity was measured as previously described. ${ }^{20}$ A detailed protocol is available in the Online Supplement.

\section{Immunohistochemistry and fluorescence microscopy}

Immunohistochemical staining was performed on cryopreserved sections of TissueTek (Agilent, CA, USA)-embedded colon carcinoma tissue (4 patients) or on paraffin-embedded atherosclerotic tissue sections, as published previously.,21 Specimens were collected according to the recommendations of the institutional ethics board including informed consent. Plaque tissue was derived from patients undergoing carotid endarterectomy (mean age $71 \pm 6.4$ years, $72 \%$ male, $27 \%$ symptomatic, $n=16$ ). 


\section{Statistics}

Sample groups were compared using a paired Student $t$-test using SPSS 21 (IBM, CA, USA). $P$-values $\leq 0.05$ were considered statistically significant. The number of individual donors per experiment is given in the figure legends. All graphs depict the mean values \pm standard deviation.

\section{Results}

We determined the expression of TF in macrophages under baseline condition as well as under polarized conditions to understand the impact of macrophages and their polarization on coagulation. When TF protein was measured in cell lysates of human macrophages, IL-4 and IL-13 significantly upregulated TF protein content whereas LPS and IFN- $\gamma$ did not change protein levels of TF compared to the levels in unpolarized macrophages (M0) (Figure 1A). Polarization conditions did not influence viability as determined by lactate dehydrogenase assay (Online Supplementary Figure S1A). TF is associated with extracellular vesicles. We, therefore, determined the TF concentration in macrophage-derived extracellular vesicles at baseline and under polarization conditions. Again, TF levels were increased under alternative polarization conditions whereas proinflammatory stimulation did not alter TF protein levels compared to the levels in microvesicles obtained from $\mathrm{M0}$ (Figure $1 \mathrm{~B})$. The main role of $\mathrm{TF}$ is the initiation of coagulation. We, therefore, analyzed the functional capacity of macrophage-derived TF-bearing microvesicles to initiate coagulation by determining the change of factor $\mathrm{X}$ to its activated form via generation of activated factor VII. Again, polarization of macrophages with IL-4 and IL13 led to a marked induction of active TF microvesicles (Figure 1C). To rule out contamination and hence lowgrade induction with LPS during alternative polarization we determined the induction of TF under alternative polarization with IL-4 and IL-13 with or without the TLR-4 inhibitor polymyxin B. Similar results were obtained in the presence and absence of polymyxin B (Online Supplementary Figure S1B). To determine whether TF protein can be detected at an early time point, we analyzed microvesicles secreted after $6 \mathrm{~h}$ for TF content. We did not observe significant changes with any polarization condition, although we did observe a non-significant increase for TF in alternatively activated macrophages (Online Supplementary Figure S1C). Besides its role in coagulation, TF has been reported to influence the migratory behavior of cells via its interaction with integrins. This cross-talk was described, among other examples, for cell migration on laminin 5 resulting in reduced migration of $\mathrm{TF}^{+}$cells. ${ }^{22}$ To analyze whether increased expression of TF reduces the formation of filopodia we analyzed filopodia formation of polarized macrophages when migrating into a laminin 5-
A

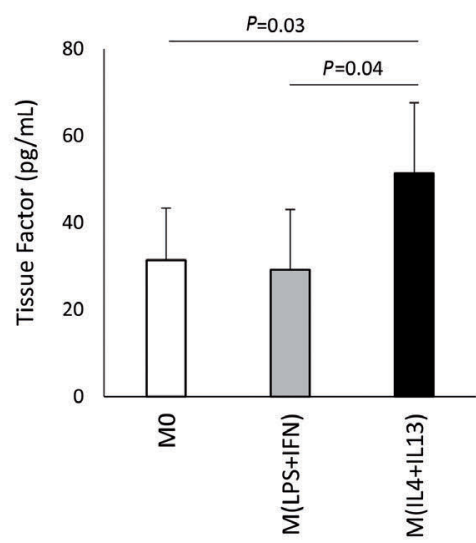

B

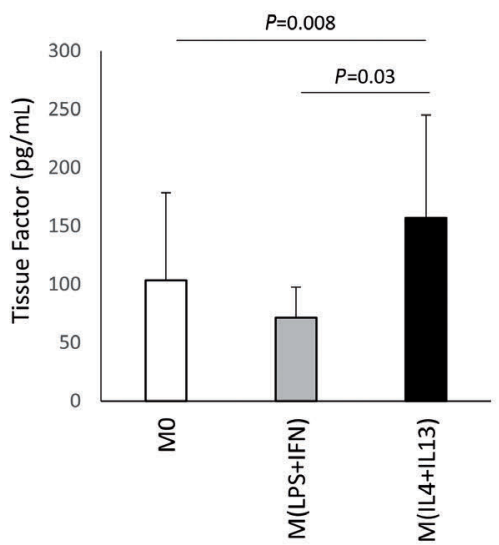

C

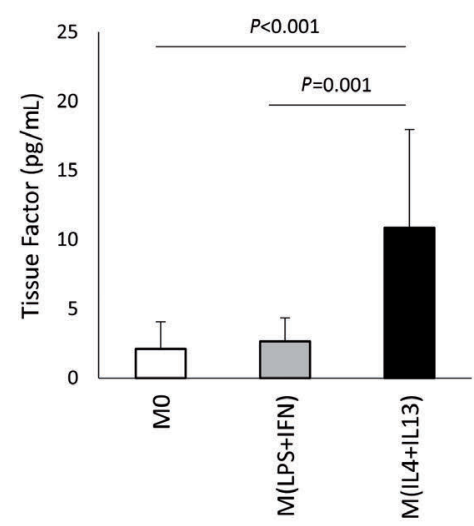

D

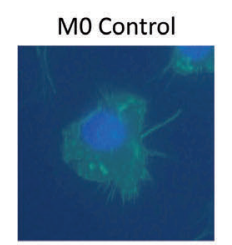

M0 Laminin 5

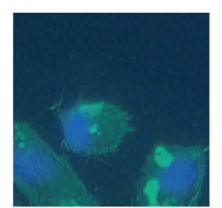

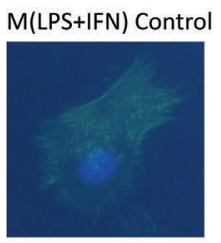

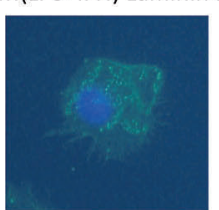

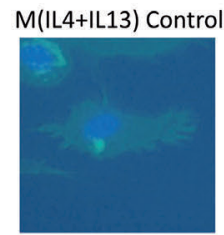

M(IL4+IL13) Laminin 5

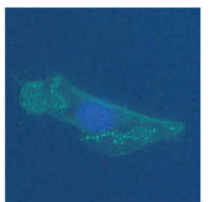

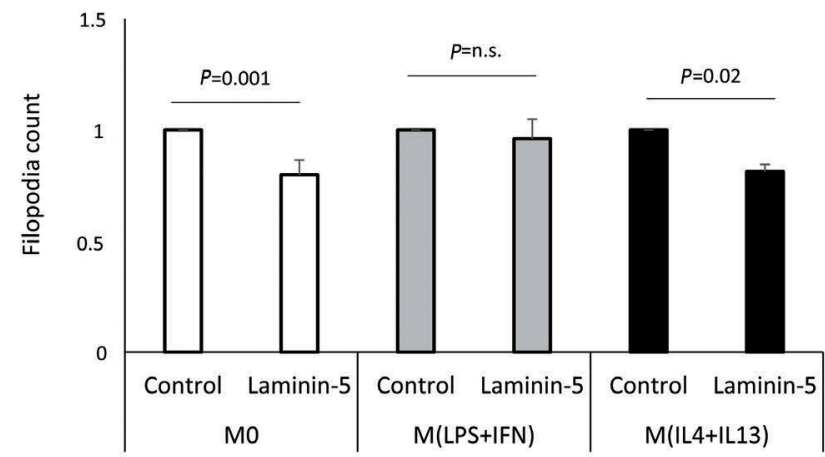

Figure 1. Tissue factor production after polarization of macrophages. (A) Tissue factor (TF) protein was determined on extracellular vesicles from supernatant ( $n=6$ ) and $(B)$ from lysed cells $(n=7)$ using a specific enzyme-linked immunosorbent assay as indicated in the Methods section. (C) TF activity on extracellular vesicles from polarized macrophages was evaluated using an activity assay as indicated in the Methods section $(n=13)$. Values are given in $\mathrm{pg} / \mathrm{mL}$ and represent mean values \pm standard deviation. (D) Capability of human polarized macrophages to form filopodia when migrating onto laminin-coated areas was evaluated by cytoskeletal staining $(n=3)$. MO: unpolarized macrophages; M(LPS+IFN): classically activated polarized macrophages; M(IL-4+IL13): alternatively activated polarized macrophages; ns: not significant. 
coated area. Our results indicate that filipodia of macrophages of classically polarized macrophages M(LPS+IFN) were not affected by the laminin 5 coating whereas in alternatively polarized macrophages, M(IL4+IL13), a significant reduction of filopodia was seen under these conditions (Figure 1D). Interestingly, the integrin described for the laminin 5 effect, namely integrin $\alpha 3$ was also downregulated in proinflammatory macrophages (Online Supplementary Figure S1D).

A significant induction of TF mRNA after IL-4 and IL-13 polarization in human macrophages was seen after $2 \mathrm{~h}$ and $6 \mathrm{~h}$ of stimulation (Figure $2 \mathrm{~A}$ ). To further control for the influence of proinflammatory polarization on TF mRNA, we also performed a time course for TF mRNA levels in $\mathrm{M}(\mathrm{LPS}+\mathrm{IFN})$. No significant regulation of TF mRNA was observed in proinflammatory macrophages at $2 \mathrm{~h}(2.9 \pm 4.2$ fold the level in $\mathrm{M0}, P=0.5$ [ $\mathrm{n}=3]), 6 \mathrm{~h}(0.9 \pm 0.4$ fold the level in $\mathrm{M} 0, P=0.8[\mathrm{n}=3])$ and $24 \mathrm{~h}(1.2 \pm 0.7$ fold the level in $\mathrm{M} 0$, $P=0.6[\mathrm{n}=3])$. The main signaling pathway observed for IL4 and IL-13 is an activation cascade dependent on signal transducer and activator of transcription (STAT) 6 signaling, as indicated by the increase of phosphorylated STAT6 in macrophages after treatment with IL-4 and IL-13 (Figure 2B). When a STAT6 inhibitor was used, the increase in TFspecific mRNA induced by IL-4 and IL-13 was completely inhibited in these cells (Figure 2C). Blocking poly ADP ribose polymerase (PARP), a possible STAT6 downstream target, also abrogated the IL-4 and IL-13-induced TF expression in these cells (Figure 2D).

Macrophages can be differentiated from monocytes via macrophage colony-stimulating factor (MCSF) or via granulocyte-macrophage colony-stimulating factor (GMCSF). Baseline extracellular vesicle TF levels were higher in GMCSF-derived macrophages than in MCSF-derived macrophages (Figure 3A). However, as can be seen from Figure $3 \mathrm{~B}$, when macrophages generated from monocytes with either MCSF or GMCSF were polarized with IL-4 and IL-13, these cells produced significantly more TF than the respective M0 macrophages and the respective macrophages polarized with LPS and IFN- $\gamma$. Polarization of macrophages to M(LPS+IFN- $\gamma$ ) and M(IL-4+IL-13) is in part reversible but has the potential to change subsequent behavior of already polarized macrophages. TF expression was again significantly induced in macrophages by IL-4 and IL-13 in both previous $\mathrm{M0}$ and $\mathrm{M}(\mathrm{LPS}+\mathrm{IFN}-\gamma)$. Interestingly, previous M(IL-4+IL-13) polarization prevented a significant increase in TF protein after restimulation (Figure 3C).

In contrast to $M(L P S+I F N-\gamma)$, monocytes treated with LPS and IFN- $\gamma$ showed significant upregulation of TF (Figure 4A). We found greater methylation around the nuclear factor-kappa B (NF- $\mathrm{B}$ ) response element within the TF promoter region in macrophages compared to monocytes obtained from the same donors (Figure 4B). When a chemical demethylating agent ${ }^{23}$ was used during polarization, TF was induced by LPS and IFN- $\gamma$ in macrophages, whereas the increase in TF was similar in macrophages polarized with IL-4 and IL-13 and the demethylating agent and in macrophages treated with only IL-4 and IL-13 (Figure 4C).

TF is associated with extracellular vesicles. To understand the effect of polarization on extracellular vesicles more globally we determined the changes in extracellular vesicle production in unpolarized and polarized macrophages. As can be seen from Figure 5A, alternative polarization of macrophages using IL-4 and IL-13 resulted in a significant increase of extracellular vesicle production over time when compared to extracellular vesicle production by unpolarized macrophages. In contrast, proinflammatory polarization of macrophages with LPS and IFN- $\gamma$ did not have a significant effect on the production of extracellular vesicles. Similar results were obtained using an ELISA, which specifically recognizes phosphatidylserine present on extracellular vesicles (Figure 5B). In addition, we used extracellular vesicle RNA as a surrogate marker for the amount of circulating vesicles. The total amount of
A

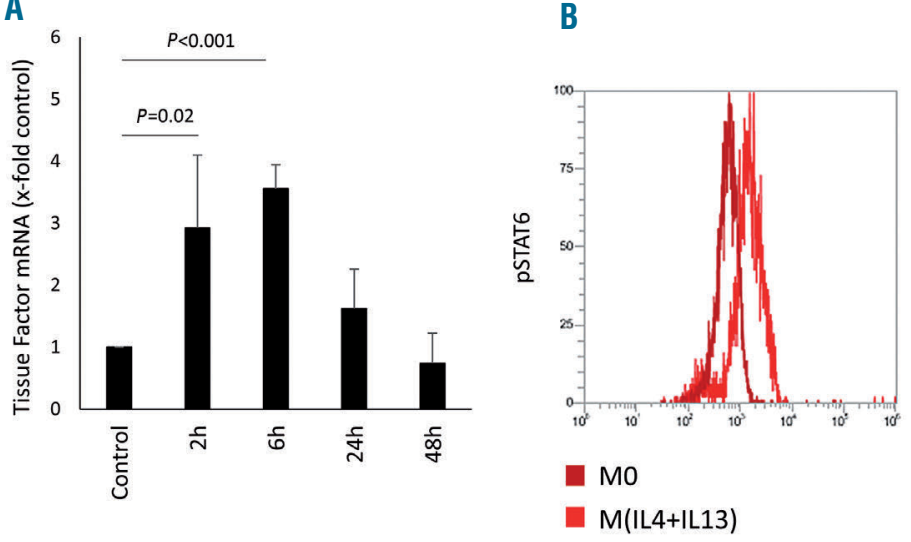

C

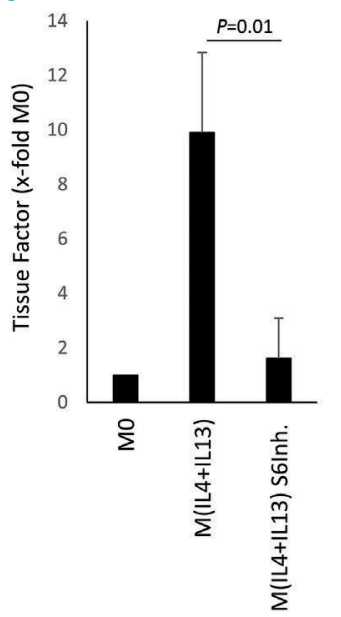

D

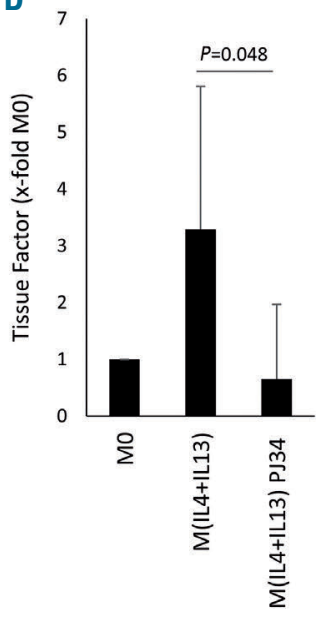

Figure 2. Tissue factor-specific mRNA induction by alternative polarization. (A) Tissue factor (TF) mRNA levels at the indicated time points in macrophages after polarization induced by interleukin (IL)-4 and IL-13 $(n=3)$. (B) Phosphorylated STAT6 as determined in macrophages 30 min after IL-4+IL-13-induced polarization in comparison to that in unpolarized MO macrophages using flow cytometry and specific antibodies as described in the Methods ( $n=3$ ). A representative image is shown. (C) TF mRNA levels in macrophages $2 \mathrm{~h}$ after IL-4+IL-13-induced polarization in the presence and absence of a specific STAT6-inhibitor (S6Inh.) at 250 $\mu \mathrm{M}$ $(n=6)$. (D) TF mRNA levels in macrophages $2 \mathrm{~h}$ after IL-4+IL-13-induced polarization in the presence and absence of the PARP-inhibitor PJ34 at 100 $\mu$ M ( $\mathrm{n}=6$ ). TF mRNA in panels A, C and D was determined by quantitative polymerase chain reaction and GAPDH was used as a housekeeping gene as indicated in the Methods section. Values are given as fold changes compared to the respective unpolarized control (MO) and represent mean values \pm standard deviation. 
RNA derived from the extracellular vesicle fraction was highest in vesicles derived from M(IL-4+IL-13) (Figure 5C). This was not, however, due to increased individual loading of extracellular vesicles with RNA, as the percentage of $\mathrm{RNA}^{+}$extracellular vesicles was similar, at around $45 \%$, for all polarization conditions: M0, M(IL-4+IL-13) and M(LPS+IFN- $\gamma$ ) (Online Supplementary Figure S2A).

pSTAT $6^{+}$macrophages were found within tumor tissue (Online Supplementary Figure S2B). Staining for TF and the macrophage marker CD206 also revealed macrophages positive for TF (Figure 6A). Not only did cells stain positive for TF but so too did the intracellular space (Figure $6 \mathrm{~B})$. These positive streaks of TF also stained positive for specks of CD206, which might indicate macrophagederived extracellular vesicles. To understand a possible link between $\mathrm{TF}^{+}$areas and areas positive for CD206 we evaluated tumor tissue from different human donors. At least seven sections from each tumor were scored for the presence of CD206 or TF. We found that especially regions with high TF expression also showed strong
A

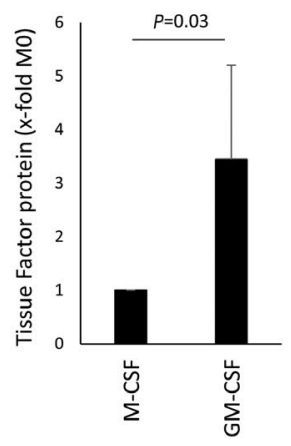

B

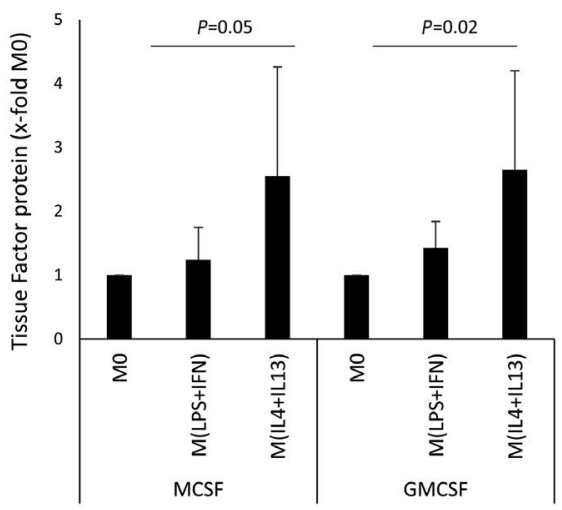

C

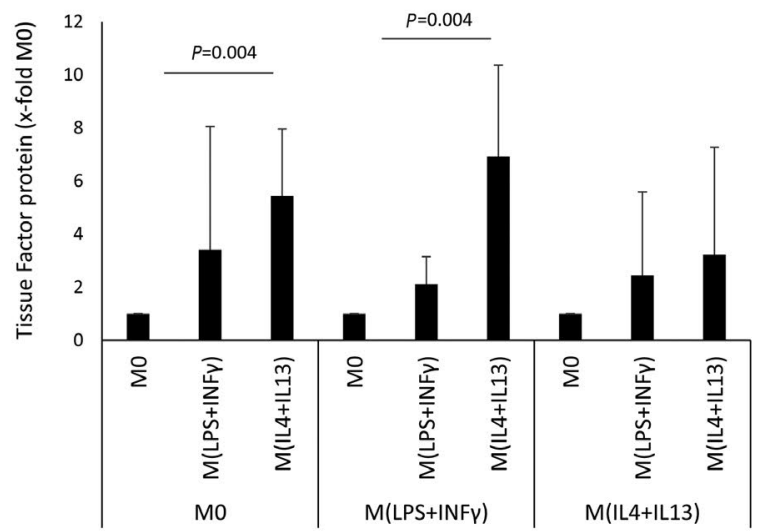

Figure 3. Tissue factor protein induction in different macrophage populations. (A) Tissue factor (TF) protein levels in supernatants from M0 macrophages generated via stimulation with macrophage colony-stimulation factor (MCSF) or granulocyte-macrophage colony-stimulating factor (GMCSF) ( $\mathrm{n}=6$ ). (B) TF protein in supernatants from macrophages that were generated either by stimulation with MCSF or GMCSF and polarized into M(LPS+IFN) and M(IL-4+IL-13) as indicated in the Methods. The respective MO was used to determine the fold changes induced by the polarization conditions $(n=6)$. (C) Macrophages were polarized for $48 \mathrm{~h}$ and afterwards repolarized for $24 \mathrm{~h}$ as indicated $(n=5)$. TF protein levels were determined using a specific enzyme-linked immunosorbent assay as indicated in the Methods section. Values are given as fold changes compared to MCSF-differentiated macrophages in panel (A) or the respective unpolarized control (M0) in panels (B) and (C) and represent mean values \pm standard deviation. MO: unpolarized macrophages; M(LPS+IFN $\gamma$ ): classically activated polarized macrophages; M(IL-4+IL13): alternatively activated polarized macrophages.

A

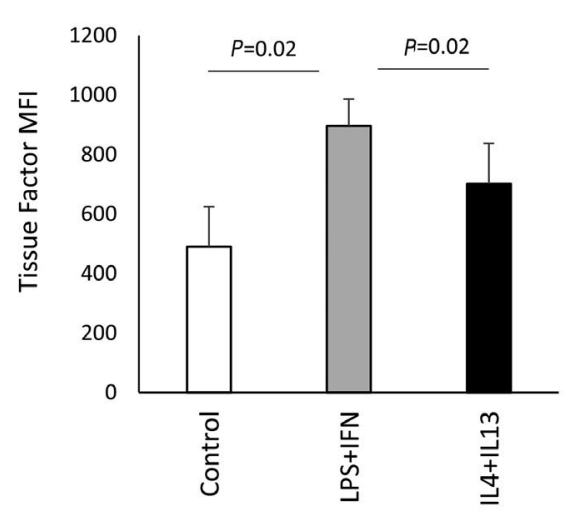

B

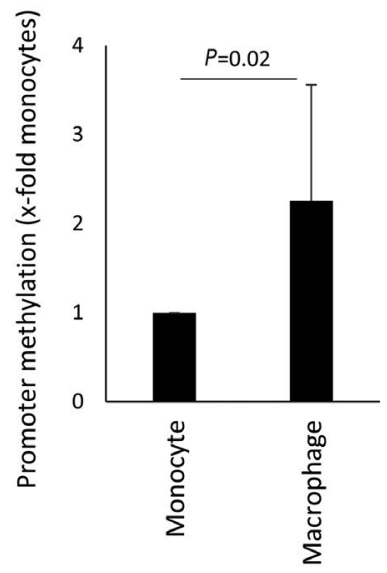

c

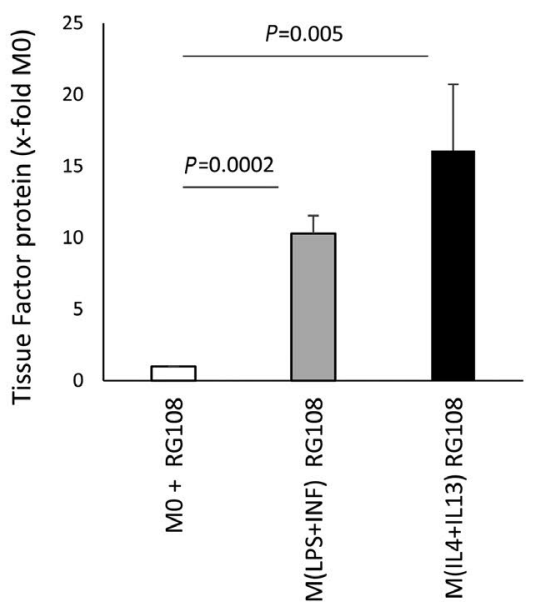

Figure 4. Epigenetic regulation of tissue factor. (A) Tissue factor (TF) expression on the surface of human monocytes cultured for $24 \mathrm{~h}$ in the presence of $100 \mathrm{ng} / \mathrm{mL}$ lipopolysaccharide (LPS) and $100 \mathrm{ng} / \mathrm{mL}$ interferon (IFN)- $\gamma$ or $20 \mathrm{ng} / \mathrm{mL}$ interleukin (IL)-4 and $20 \mathrm{ng} / \mathrm{mL}$ IL-13 or without any addition (control) was analyzed by flow cytometry using a specific antibody as described in the Methods. Data are shown as mean fluorescence intensity (MFI) ( $n=4)$. (B) Methylation of the TF-promoter was analyzed by quantitative polymerase chain reaction as indicated in the Methods section. Macrophage values are given as fold increases compared to the monocyte value, which was set at 1 . Monocytes and macrophages from the same individuals were compared $(n=4)$. (C) TF protein was determined using a specific enzymelinked immunosorbent assay as indicated in the Methods section in the presence of the demethylating agent RG108 at a concentration of $5 \mu \mathrm{M}$ during polarization. Values are given as fold changes compared to the respective unpolarized control $(M 0)(n=3)$ and represent mean values \pm standard deviation. MFl: mean fluorescence intensity; MO: unpolarized macrophages; M(LPS+IFN): classically activated polarized macrophages; M(IL-4+IL13): alternatively activated polarized macrophages. 
CD206 expression and regions with low TF expression were predominantly negative or low for CD206 (Figure 6C). Besides tumors, atherosclerotic plaques also contain areas of high TF expression. ${ }^{10} \mathrm{We}$, therefore, analyzed the presence of $\mathrm{TF}$ in $\mathrm{CD}^{206^{+}}$regions or $\mathrm{CD} 80^{+}$regions within human atherosclerotic lesions. We were able to detect TF in both $\mathrm{CD}_{206^{+}}$regions and in $\mathrm{CD} 80^{+}$regions. However, adjusted TF intensity was higher in regions positive for CD206, indicating an association of increased TF presence close to alternatively activated macrophages (Figure 6D). Furthermore, using flow cytometry, we analyzed macrophages isolated from atherosclerotic plaques from $\mathrm{ApoE}^{-/}$mice fed a high fat diet for 20 weeks. The gating strategy is shown in Online Supplementary Figure S3. Overall, proinflammatory CD80 high macrophages were less positive for TF surface expression as compared to CD206 $6^{\text {high }}$ macrophages (Figure 6E). Furthermore, CD206 $6^{\text {high }}$ macrophages had a two-fold higher TF surface intensity staining as determined by a comparison of mean fluorescence intensity of
CD206 ${ }^{\text {high }}$ macrophages and CD80 high macrophages (Figure 6F).

\section{Discussion}

Based on extensive experimental work and clinical data it has been well established that circulating monocytes are an important source of TF and, that by expressing this major component of coagulation, they play a key role in linking inflammation and thrombosis in various pathologies. ${ }^{10}$ Much less is known about the expression of TF and its regulation in macrophages. Here, for the first time we provide evidence that in human macrophages the expression of TF is not altered in a proinflammatory environment but is significantly enhanced when these cells are alternatively polarized. When human macrophages were polarized with IL-4 and IL-13 a significant increase in mRNA specific for TF was observed after $2 \mathrm{~h}$ and $6 \mathrm{~h}$. Alternative polarization also caused a significant increase in TF protein
A

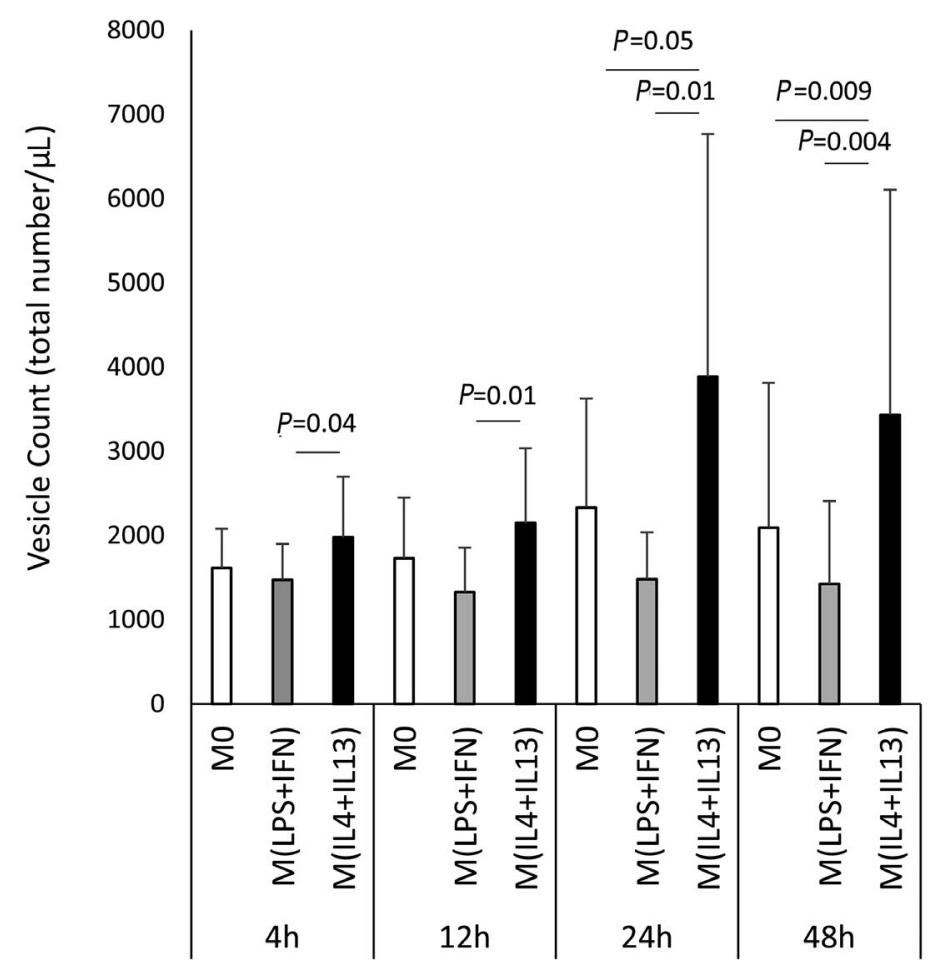

B

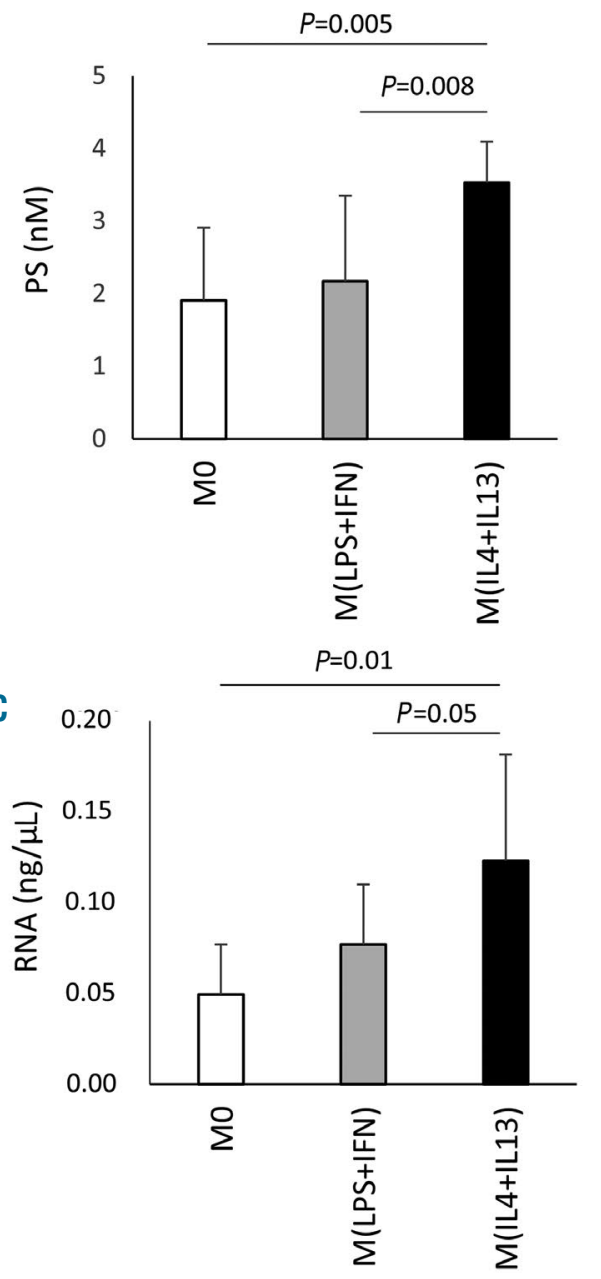

Figure 5. Influence of polarization conditions on extracellular vesicle production of human macrophages. (A) Numbers of extracellular vesicles in the supernatant of unpolarized macrophages and macrophages polarized in the presence of $100 \mathrm{ng} / \mathrm{mL}$ lipopolysaccharide (LPS) and $100 \mathrm{ng} / \mathrm{mL}$ interferon (IFN)- $\gamma$ or $20 \mathrm{ng} / \mathrm{mL}$ interleukin (IL)-4 and $20 \mathrm{ng} / \mathrm{mL}$ IL-13 were determined by flow cytometry as indicated in the Methods section. Values are given as total vesicle count per $\mu \mathrm{L}$ : $\mathrm{n}=9$ for $4 \mathrm{~h}$ and $12 \mathrm{~h} ; \mathrm{n}=12$ for $24 \mathrm{~h}$; and $\mathrm{n}=14$ for $48 \mathrm{~h}$. (B) Phosphatidylserine (PS) content of extracellular vesicles in the supernatant of M0 and macrophages polarized in the presence of $100 \mathrm{ng} / \mathrm{mL}$ LPS and $100 \mathrm{ng} / \mathrm{mL}$ IFN- $\gamma$ or $20 \mathrm{ng} / \mathrm{mL} \mathrm{IL-4}$ and $20 \mathrm{ng} / \mathrm{mL} \mathrm{IL-13}$ for $48 \mathrm{~h}$ was determined using a specific enzyme-linked immunosorbent assay as indicated in the Methods. Values are given in nM PS $(n=6)$. (C) Total extracellular vesicle-derived RNA was evaluated in the supernatant of MO and macrophages polarized in the presence of $100 \mathrm{ng} / \mathrm{mL}$ LPS and $100 \mathrm{ng} / \mathrm{mL}$ IFN- $\gamma$ or $20 \mathrm{ng} / \mathrm{mL}$ IL-4 and $20 \mathrm{ng} / \mathrm{mL}$ IL-13 for $48 \mathrm{~h}$ as indicated in the Methods section. Values are given in $n g / \mu \mathrm{L}$ RNA $(n=5)$ and represent mean values \pm standard deviation. M0: unpolarized macrophages; M(LPS+IFN): classically activated polarized macrophages; M(IL-4+IL13): alternatively activated polarized macrophages. 
in such cells. In contrast, TF production in macrophages was not affected by inflammatory polarization with LPS and IFN- $\gamma$.

Differentiation of monocytes to macrophages can be induced by MCSF or GMCSF to simulate a chronic inflammatory state or an acute event, respectively. ${ }^{24,25}$ Both cytokines were reported to result in differentiation of monocytes into mature macrophages, although GMCSFderived macrophages display a higher proinflammatory potential. ${ }^{24}$ Here we show that human macrophages generated from monocytes by stimulation with GMCSF produce significantly more TF than macrophages generated from monocytes by stimulation with MCSF. We hypothesize that the increased proinflammatory state induced by GMCSF might be responsible for this higher basal TF protein level in GMCSF-derived macrophages as compared to their MCSF-derived counterparts. However, a significant increase in TF after alternative polarization was evident for macrophages differentiated from monocytes by either MCSF or GMCSF. Thus, this increase in TF was not dependent on the initial monocyte to macrophage differentiation by these colony-stimulating factors.

Besides coagulation, TF might also modulate the migra- tory capacity of macrophages in a way similar to that observed for other cell types..22 Several integrins have already been reported to interact with $\mathrm{TF}^{26}$ Interestingly, integrin $\alpha 4$ was associated with $\mathrm{TF}^{+}$microvesicles shed from macrophages. ${ }^{27}$ This proinflammatory shedding was dependent on activation of caspase-1. ${ }^{28}$ Integrin $\alpha 3$ was associated with TF-dependent migration in keratinocytes. ${ }^{22}$ Our results indicate that integrin $\alpha 3$ is downregulated with polarization. We speculate that low levels of both integrin $\alpha 3$ and TF in proinflammatory macrophages might be responsible for the ability to form filopodia in laminin 5positive areas.

Macrophages within inflammatory tissues or within tumors are derived from differentiated monocytes. ${ }^{29}$ Signals encountered in the microenvironment have the potential to specifically shape the developing macrophages..$^{30}$ Previous results demonstrated that stimulating macrophages with pro- or anti-inflammatory cytokines leads to opposing transcriptional functional programs and results in silencing or activation of genes causing a memory function of initial polarization conditions. . $^{31,32}$ Previously, we were able to demonstrate that an initial alternative activation of macrophages led to a subsequent
A

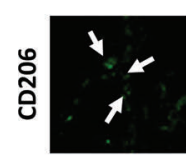

হ̄
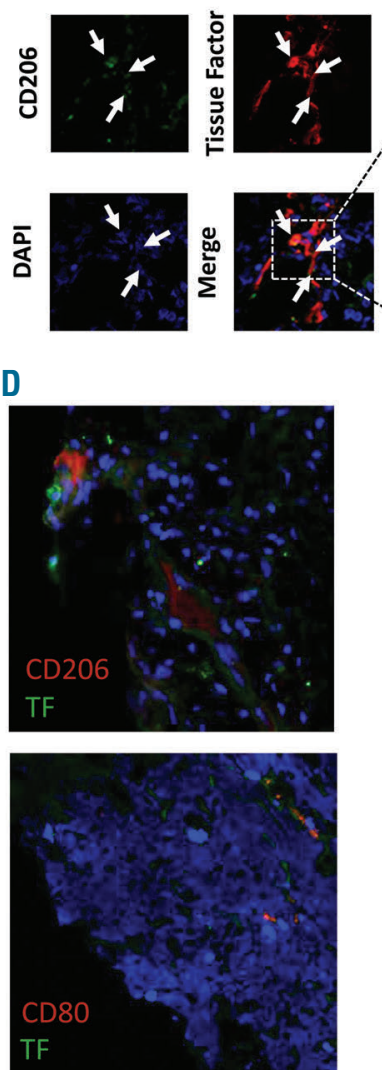

B
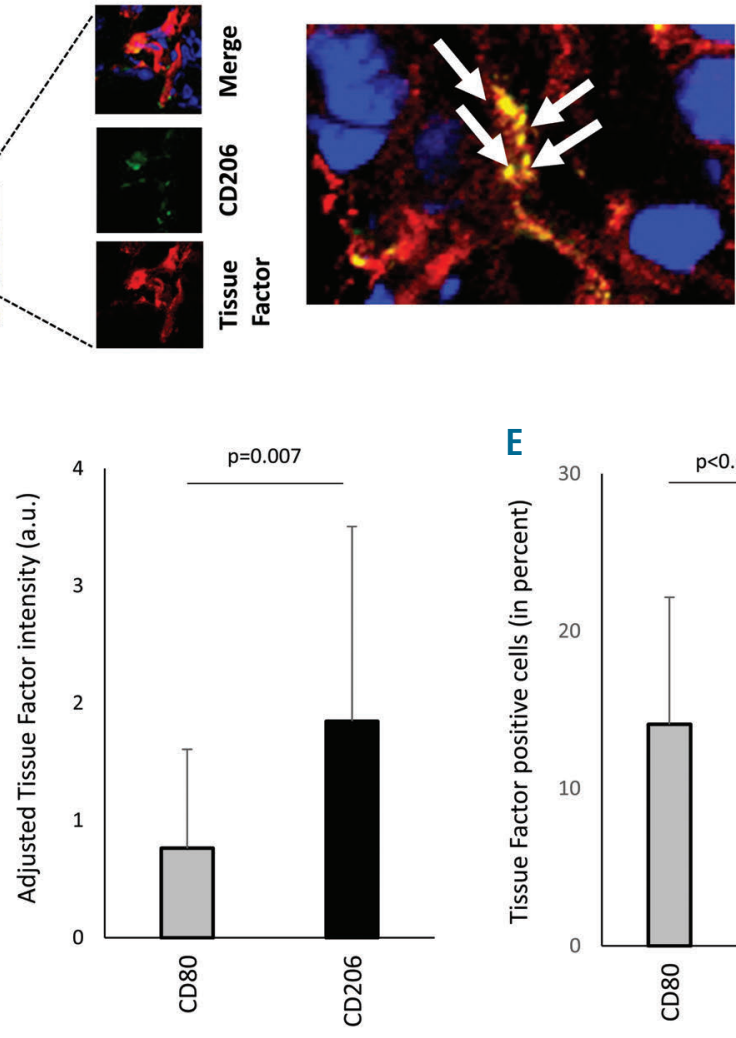

E
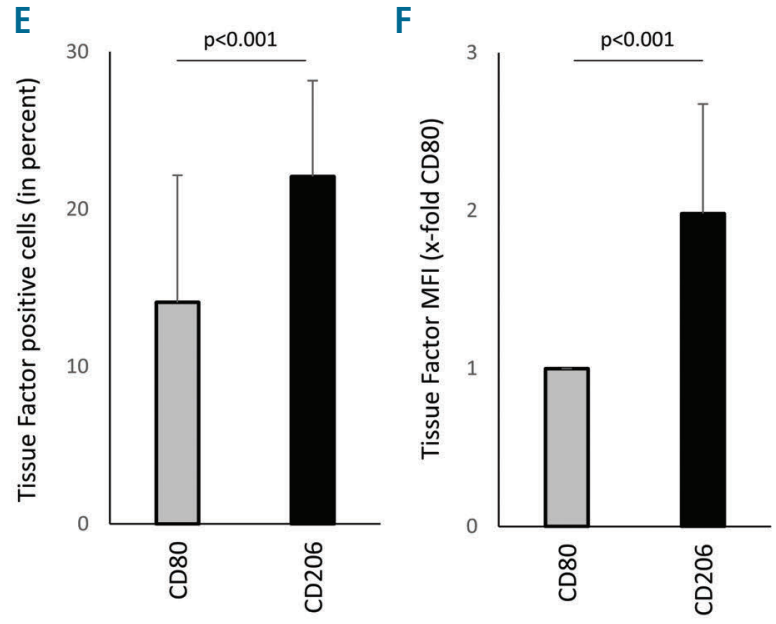

Figure 6. Staining of tissue factor-positive macrophages in sections of colon carcinoma. (A) Tissue factor (TF) (red) and CD206 (green) were stained in colon cancer tissue using specific antibodies as described in the Methods. CD206 macrophages positive for TF are indicated with white arrows. The boxed area is shown in more detail. (B) White arrows indicate acellular regions that showed double staining for CD206 and TF (orange), which could represent extracellular vesicles derived from macrophages positive for CD206 and TF. (C) Distribution of CD206 macrophages was evaluated and scored in areas with low, medium, and high TF density. (D) Human atherosclerotic plaque tissue was stained for TF (green) and either CD206 (red) alternatively activated macrophages or CD80 (red) for proinflammatory macrophages. Adjusted TF intensity to macrophage intensity demonstrated an increase in TF in $\mathrm{CD}^{206^{+}}$regions. Values are given as adjusted tissue factor intensity (arbitrary units) mean values \pm standard deviation (SD) $(n=16$ patients). (E, F) Mouse macrophages from atherosclerotic plaques were isolated as indicated in the Online Supplement. Proinflammatory macrophages were less positive for TF and showed reduced mean fluorescence intensity compared to alternatively activated CD206 macrophages $(n=11)$. Values represent mean values \pm SD. DAPI: 4',6-diamidino-2-phenylindole; a.u.: arbitrary units. 
activation of the plasminogen activator inhibitor-1 promoter for inflammatory induction. 'Here we show that TF inducibility with IL-4 and IL-13 was reduced after prior alternative polarization. However, prior inflammatory polarization did not alter the capability of IL-4 and IL-13 to induce TF. Previous reports suggested a dysfunctional mitochondrial phenotype after proinflammatory polarization resulting in an altered capability of IL-4 to induce its target genes and preventing macrophage repolarization. ${ }^{33}$ Our results suggest that TF is an alternative activationdependent target gene that is not affected by changes in mitochondrial function as TF production could still be induced after alternative polarization of previously proinflammatory polarized macrophages. Furthermore, TF release seems to be delayed and in parallel to microvesicle release as both TF amount and microvesicle number were significantly different in alternatively polarized macrophages $24 \mathrm{~h}$ after polarization.

In monocytes TF was shown to be induced by NF-kB and AP-1-dependent signaling. ${ }^{34}$ In murine macrophages an induction of TF was demonstrated after LPS stimulation. ${ }^{35}$ Human cells were also reported to retain the capacity to react to LPS or IFN- $\gamma$ for a certain time while differentiating from monocytes to macrophages. ${ }^{36}$ In addition to NF- $\kappa \mathrm{B}$ and AP-1, the TF promoter contains Sp1 binding sites ${ }^{37}$ and is enhanced by Pin $1 .^{38}$ Furthermore, in mouse macrophages loss of PARP14 was demonstrated to increase the levels of TF expression. ${ }^{39}$ In addition, PARP14 is in part regulated by STAT6 as STAT6 activation induces a switch in PARP14 from a repressor to a promoter of STAT6 signaling via the ribosylation of histone deacetylases previously recruited to IL-4 response elements..$^{40}$ Of note, STAT6 is the main downstream target activated by IL-4 and IL-13 signaling. ${ }^{41}$ In the present work we demonstrate that inhibition of either STAT6 or PARP activity abolished the induction of TF mRNA in macrophages by IL-4 and IL-13. Our findings suggest that macrophages upregulate TF in vivo in a STAT6inducing environment and that TF might be a marker protein for STAT6 signaling and, therefore, alternative polarization in macrophages. Nonetheless, it must be emphasized that macrophages in general express TF already at baseline.

There is ample evidence to support the notion that monocytes are an important source of circulating TF. At resting conditions already around $1.5 \%$ of $\mathrm{CD}_{14} 4^{+}$monocytes are positive for $\mathrm{TF}^{42}$ When monocytes are exposed to LPS TF expression is quickly and transiently upregulated in these cells. ${ }^{43,44}$ We have identified in our study a difference of TF induction between monocytes and macrophages as our results indicate that human macrophages do not react to LPS treatment with upregulation of TF but display increased TF production only after alternative polarization. This is somehow in contrast to earlier reports that suggested a similar behavior of monocytes and macrophages in response to defined stimuli. ${ }^{45,46}$ However, whereas we used MCSF or GMCSF for macrophage maturation those studies were performed on macrophages derived from monocytes in the presence of human serum without a defined macrophage maturation factor suggesting that the cells studied could be monocytes still in transition to macrophages.

Epigenetic changes within a promoter region hold the potential to alter the response of cells to certain stimuli. ${ }^{47}$ We, therefore, determined the methylation state around the NF- $\mathrm{BB}$ response element, which is responsible for reacting to LPS signaling, in monocytes and macrophages derived from the same donor and observed a higher grade of methylation of the TF promoter in macrophages as compared to the respective monocytes from the same donor. Interestingly, addition of a demethylating agent restored the capacity of macrophages to react to LPS stimulation with upregulation of TF. We, therefore, hypothesize that the difference between monocytes and macrophages in their respective ability to react towards LPS with an increase in TF could be due to epigenetic changes within the TF promoter.

Extracellular vesicles were identified because of their ability to support coagulation. ${ }^{48}$ Among such vesicles, TFbearing ones were demonstrated to propagate thrombus formation. ${ }^{49}$ Whereas monocyte-derived, TF-positive extracellular vesicles are common in the circulation ${ }^{50}$ and monocyte-derived extracellular vesicles are associated with several pathological states including cardiovascular disease and inflammatory disorders ${ }^{51}$ little is known about the production of TF-positive extracellular vesicles derived from macrophages. Our results indicate that macrophages show a basal production of extracellular vesicles, which can be increased by alternative activation. In addition, we found that these extracellular vesicles shed from macrophages are phosphatidylserine positive. It should be emphasized that TF it is not only regulated at the level of mRNA expression but also at the post-translational level with phosphatidylserine converting TF from a cryptic to a decrypted prothrombotic form..$^{10,52,53}$ Our findings described above therefore support the notion that macrophagederived vesicles might have the capacity to activate TF. We also show here that alternative polarization increased both the total amount of extracellular vesicles as well as the amount of TF on these extracellular vesicles significantly as compared to vesicles from unpolarized macrophages or macrophages that had undergone proinflammatory polarization.

Finally, our in vitro data showing that alternatively activated macrophages produce TF were supported by immunohistochemical analysis of colon carcinoma sections. As alternative polarization is most common in tumors, we analyzed histochemical sections of colon carcinoma, a tumor which has already been shown to have increased IL-4 expression ${ }^{54}$ and contain $\mathrm{TF}^{+}$cells. ${ }^{55}$ To identify proinflammatory macrophages we used CD80, whereas alternatively activated macrophages were identified by CD206. Tumor-associated macrophages have already been associated with IL-4 activation. ${ }^{56}$ Here we were able to identify STAT6 ${ }^{+}$macrophages within these sections of colon carcinoma, indicating IL-4 and IL-13 signaling. Furthermore, macrophages within these sections stained positive for CD206, an established marker for alternatively polarized macrophages, and for TF. We also showed that extracellular TF in part co-localized with extracellular CD206 possibly suggesting the presence of macrophagederived extracellular vesicles in these sections. To understand a possible link between $\mathrm{TF}^{+}$areas and areas positive for CD206 we evaluated tumor tissue from four different human donors. At least seven sections from each tumor were scored for the presence of CD206 or TF. We found that especially regions with high expression of TF also had high expression of CD206 and regions with low TF expression were predominantly negative or low for CD206. Besides tumor tissue, atherosclerotic plaques have also been demonstrated to contain TF with TF protein localization associated with plaque macrophages. ${ }^{57}$ Interestingly, 
already back in 1989, Wilcox et al. demonstrated that not all TF was associated with cells, ${ }^{57}$ a notion our data from both atherosclerotic tissue and cancer tissue would support. Within the atherosclerotic environment macrophages are polarized into different subsets. Both proinflammatory and alternatively activated macrophages were reported to coexist within this environment. ${ }^{58}$ Our data demonstrate that both proinflammatory $\mathrm{CD} 80^{+}$macrophages as well as alternatively activated $\mathrm{CD} 206^{+}$macrophages are sources for TF. Nonetheless, CD206 ${ }^{+}$macrophages are associated with an increased production of TF within the atherosclerotic lesion, supporting our in vitro data showing induction of TF through alternative activation.

In conclusion, we provide new evidence that alternative polarization of macrophages leads to a procoagulatory phenotype through the expression of TF and the production of TF-containing extracellular vesicles and might change overall cellular behavior including migratory preferences. Together with our previously published results showing that alternatively activated macrophages express increased levels of the antifibrinolytic serpin plasminogen activator inhibitor- $1,{ }^{7}$ our results suggest that alternatively polarized macrophages support thrombus formation and suppress thrombolysis ${ }^{59}$ and thus represent a novel cellular mediator linking macrophages and thrombosis in pathologies characterized by these events.

\section{Disclosures}

This work was supported by a grant from the FWF, the Austrian Science Fund to JW (SFB-54).

\section{Contributions}

PJH, JM, JK, JKP, BT, CK, LH, PH, MM, SS and KW performed the research; JKPWSS analyzed the data; PJH and JW wrote the manuscript; JAS, SD, MBF, KH, CH, RO, IP and JW supervised the study.

\section{Acknowledgments}

This work was supported by a grant from the FWF, the Austrian Science Fund, to IP, JS and JW (SFB-54).

\section{References}

1. Glass CK. Genetic and genomic approaches to understanding macrophage identity and function. Arterioscler Thromb Vasc Biol. 2015;35(4):755-762.

2. Cybulsky MI, Cheong C, Robbins CS. Macrophages and dendritic cells: partners in atherogenesis. Circ Res. 2016;118(4):637-652.

3. Murray PJ, Wynn TA. Protective and pathogenic functions of macrophage subsets. Nat Rev Immunol. 2011;11(11):723-737.

4. Murray PJ. Macrophage polarization. Annu Rev Physiol. 2017;79:541-66.

5. Lech M, Anders HJ. Macrophages and fibrosis: how resident and infiltrating mononuclear phagocytes orchestrate all phases of tissue injury and repair. Biochim Biophys Acta. 2013;1832(7):989-997.

6. Porta C, Rimoldi M, Raes G, et al. Tolerance and M2 (alternative) macrophage polarization are related processes orchestrated by p50 nuclear factor kappaB. Proc Natl Acad Sci U S A. 2009:106(35):14978-14983.

7. Hohensinner PJ, Baumgartner J, KralPointner JB, et al. PAI-1 (plasminogen activator inhibitor-1) expression renders alternatively activated human macrophages proteolytically quiescent. Arterioscler Thromb Vasc Biol. 2017:37 (10):1913-1922.

8. Mantovani A, Sozzani S, Locati M, Allavena P, Sica A. Macrophage polarization: tumorassociated macrophages as a paradigm for polarized M2 mononuclear phagocytes. Trends Immunol. 2002;23(11):549-555.

9. Sica A, Mantovani A. Macrophage plasticity and polarization: in vivo veritas. J Clin Invest. 2012;122(3):787-795.

10. Grover SP, Mackman N. Tissue factor: an essential mdiator of hemostasis and trigger of thrombosis. Arterioscler Thromb Vasc Biol. 2018:38(4):709-725.

11. Pawlinski R, Wang JG, Owens AP 3rd, et al. Hematopoietic and nonhematopoietic cell tissue factor activates the coagulation cascade in endotoxemic mice. Blood. 2010;116(5):806-814

12. Berckmans RJ, Sturk A, van Tienen LM, Schaap MC, Nieuwland R. Cell-derived vesicles exposing coagulant tissue factor in saliva. Blood. 2011:117(11):3172-3180.
13. Gardiner C, Harrison P, Belting M, et al. Extracellular vesicles, tissue factor, cancer and thrombosis - discussion themes of the ISEV 2014 Educational Day. J Extracell Vesicles. 2015;4:26901.

14. Butler JT, Abdelhamed S, Kurre P. Extracellular vesicles in the hematopoietic microenvironment. Haematologica. 2018; 103(3):382-394

15. Ettelaie C, Collier ME, Maraveyas A, Ettelaie R. Characterization of physical properties of tissue factor-containing microvesicles and a comparison of ultracentrifuge-based recovery procedures. J Extracell Vesicles. 2014;3.

16. Yanez-Mo M, Siljander PR, Andreu Z, et al. Biological properties of extracellular vesicles and their physiological functions. J Extracell Vesicles. 2015:4:27066.

17. Tripisciano C, Weiss R, Eichhorn T, et al. Different potential of extracellular vesicles to support thrombin generation: contributions of phosphatidylserine, tissue factor, and cellular origin. Sci Rep. 2017;7(1):6522.

18. Hohensinner PJ, Baumgartner J, Ebenbauer $B$, et al. Statin treatment reduces matrix degradation capacity of proinflammatory polarized macrophages. Vascul Pharmacol. 2018;110:49-54

19. Thaler B, Hohensinner PJ, Krychtiuk KA, et al. Differential in vivo activation of monocyte subsets during low-grade inflammation through experimental endotoxemia in humans. Sci Rep. 2016;6:30162.

20. Khorana AA, Francis CW, Menzies KE, et al. Plasma tissue factor may be predictive of venous thromboembolism in pancreatic cancer. J Thromb Haemost. 2008;6(11): 1983-1985.

21. Hohensinner PJ, Takacs N, Kaun C, et al. Urokinase plasminogen activator protects cardiac myocytes from oxidative damage and apoptosis via hOGG1 induction. Apoptosis. 2017:22(8):1048-1055

22. Dorfleutner A, Hintermann E, Tarui T, Takada Y, Ruf W. Cross-talk of integrin alpha3beta1 and tissue factor in cell migration. Mol Biol Cell. 2004;15(10):4416-4425.

23. Brueckner B, Garcia Boy R, Siedlecki P, et al. Epigenetic reactivation of tumor suppressor genes by a novel small-molecule inhibitor of human DNA methyltransferases. Cancer
Res. 2005;65(14):6305-6311.

24. Hamilton JA. Colony-stimulating factors in inflammation and autoimmunity. Nat Rev Immunol. 2008:8(7):533-544

25. Hamilton TA, Zhao C, Pavicic PG, Jr., Datta S. Myeloid colony-stimulating factors as regulators of macrophage polarization. Front Immunol. 2014;5:554.

26. Kocaturk B, Versteeg HH. Tissue factor-integrin interactions in cancer and thrombosis: every Jack has his Jill. J Thromb Haemost. 2013;11 Suppl 1:285-293

27. Rothmeier AS, Marchese P, Langer F, et al. Tissue factor prothrombotic activity is regulated by integrin-arf6 trafficking Arterioscler Thromb Vasc Biol. 2017;37(7): 1323-1331.

28. Rothmeier AS, Marchese P, Petrich BG, et al. Caspase-1-mediated pathway promotes generation of thromboinflammatory microparticles. J Clin Invest. 2015;125 (4):1471-1484.

29. Wynn TA, Chawla A, Pollard JW. Macrophage biology in development, homeostasis and disease. Nature. 2013;496 (7446):445-455

30. Hoeksema MA, Glass CK. Nature and nurture of tissue-specific macrophage phenotypes. Atherosclerosis. 2019;281:159-167.

31. Czimmerer Z, Daniel B, Horvath A, et al. The transcription factor STAT6 mediates direct repression of inflammatory enhancers and Imits activation of alternatively polarized macrophages. Immunity. 2018;48(1): 75-90 e6.

32. Qiao Y, Kang K, Giannopoulou E, Fang C, Ivashkiv LB. IFN- $\gamma$ induces histone 3 lysine 27 trimethylation in a small subset of promoters to stably silence gene expression in human macrophages. Cell Rep. 2016;16(12): 3121-3129.

33. Van den Bossche J, Baardman J, Otto NA, et al. Mitochondrial dysfunction prevents repolarization of inflammatory macrophages. Cell Rep. 2016;17(3):684-696.

34. Mackman N, Brand K, Edgington TS. Lipopolysaccharide-mediated transcriptional activation of the human tissue factor gene in THP-1 monocytic cells requires both activator protein 1 and nuclear factor kappa B binding sites. J Exp Med. 1991;174(6):15171526 
35. Ahamed J, Niessen F, Kurokawa T, et al. Regulation of macrophage procoagulant responses by the tissue factor cytoplasmic domain in endotoxemia. Blood. 2007;109 (12):5251-5259.

36. Scheibenbogen C, Moser H, Krause S, Andreesen R. Interferon-gamma-induced expression of tissue factor activity during human monocyte to macrophage maturation. Haemostasis. 1992;22(4):173-178.

37. Moll T, Czyz M, Holzmuller $\mathrm{H}$, et al. Regulation of the tissue factor promoter in endothelial cells. Binding of NFkB-, AP-1and Sp1-like transcription factors. J Biol Chem. 1995;270(8):3849-3857.

38. Kurakula K, Koenis DS, Herzik MA Jr, et al. Structural and cellular mechanisms of peptidyl-prolyl isomerase Pin1-mediated enhancement of tissue factor gene expression, protein half-life, and pro-coagulant activity. Haematologica. 2018;103(6):10731082.

39. Iqbal MB, Johns $M$, Cao J, et al. PARP-14 combines with tristetraprolin in the selective posttranscriptional control of macrophage tissue factor expression. Blood. 2014;124(24):3646-3655.

40. Mehrotra P, Riley JP, Patel R, Li F, Voss L, Goenka S. PARP-14 functions as a transcriptional switch for Stat6-dependent gene activation. J Biol Chem. 2011;286(3):1767-1776.

41. Goenka S, Kaplan MH. Transcriptional regulation by STAT6. Immunol Res. 2011;50(1):87-96.

42. Egorina EM, Sovershaev MA, Bjorkoy G, et al. Intracellular and surface distribution of monocyte tissue factor: application to inter- subject variability. Arterioscler Thromb Vasc Biol. 2005;25(7):1493-1498.

43. Franco RF, de Jonge E, Dekkers PE, et al. The in vivo kinetics of tissue factor messenger RNA expression during human endotoxemia: relationship with activation of coagulation. Blood. 2000;96(2):554-559.

44. Gregory SA, Morrissey JH, Edgington TS. Regulation of tissue factor gene expression in the monocyte procoagulant response to endotoxin. Mol Cell Biol. 1989;9(6):27522755.

45. Miserez R, Jungi TW. LPS-induced, but not interferon-gamma-induced procoagulant activity of suspended human macrophages is followed by a refractory state of low procoagulant expression. Thromb Res. 1992;65(6):733-744.

46. Schwager I, Jungi TW. Effect of human recombinant cytokines on the induction of macrophage procoagulant activity. Blood. 1994:83(1):152-160

47. Jaenisch R, Bird A. Epigenetic regulation of gene expression: how the genome integrates intrinsic and environmental signals. Nat Genet. 2003;33 Suppl:245-254.

48. Wolf P. Nature and significance of platelet products in human plasma. Br J Haematol. 1967;13(3):269-288.

49. Key NS. Analysis of tissue factor positive microparticles. Thromb Res. 2010;125 Suppl 1:S42-S45

50. Angelillo-Scherrer A. Leukocyte-derived microparticles in vascular homeostasis. Circ Res. 2012;110(2):356-369.

51. Suades R, Padro T, Badimon L. The role of blood-borne microparticles in inflammation and hemostasis. Semin Thromb Hemost. 2015;41(6):590-606

52. Bach RR, Moldow CF. Mechanism of tissue factor activation on HL-60 cells. Blood. 1997;89(9):3270-3276.

53. Zelaya H, Rothmeier AS, Ruf W. Tissue factor at the crossroad of coagulation and cell signaling. J Thromb Haemost. 2018;16(10): 1941-1952.

54. Baier PK, Wolff-Vorbeck G, Eggstein S, Baumgartner U, Hopt UT. Cytokine expression in colon carcinoma. Anticancer Res. 2005;25(3B):2135-2139.

55. Eriksson O, Asplund A, Hegde G, et al. A stromal cell population in the large intestine identified by tissue factor expression that is lost during colorectal cancer progression. Thromb Haemost. 2016;116(6):1050-1059.

56. Wang HW, Joyce JA. Alternative activation of tumor-associated macrophages by IL-4 priming for protumoral functions. Cell Cycle. 2010;9(24):4824-4835.

57. Wilcox JN, Smith KM, Schwartz SM Gordon D. Localization of tissue factor in the normal vessel wall and in the atherosclerotic plaque. Proc Natl Acad Sci U S A 1989;86(8):2839-2843.

58. Stoger JL, Gijbels MJ, van der Velden S, et al Distribution of macrophage polarization markers in human atherosclerosis. Atherosclerosis. 2012;225(2):461-468.

59. Semeraro F, Ammollo CT, Semeraro N Colucci $\mathrm{M}$. Tissue factor-expressing monocytes inhibit fibrinolysis through a TAFImediated mechanism, and make clots resistant to heparins. Haematologica. 2009:94(6):819-826. 